\title{
Resonant enhancement of the Fulde-Ferell-Larkin-Ovchinnikov state in three dimensions by a one-dimensional optical potential
}

\author{
Jeroen P. A. Devreese, ${ }^{1}$ Sergei N. Klimin,,${ }^{1, *}$ and Jacques Tempere ${ }^{1,2}$ \\ ${ }^{1}$ Theorie van Kwantumsystemen en Complexe Systemen (TQC), Universiteit Antwerpen, B-2020 Antwerpen, Belgium \\ ${ }^{2}$ Lyman Laboratory of Physics, Harvard University, Cambridge, Massachusetts 02138, USA
}

(Received 27 August 2010; published 14 January 2011)

\begin{abstract}
We describe an imbalanced superfluid Fermi gas in three dimensions within the path-integral framework. To allow for the formation of the Fulde-Ferell-Larkin-Ovchinnikov state (FFLO state), a suitable form of the saddle point is chosen, in which the pairs have a finite center-of-mass momentum. To test the correctness of this path-integral description, the zero-temperature phase diagram for an imbalanced Fermi gas in three dimensions is calculated, and compared to recent theoretical results. Subsequently, we investigate two models that describe the effect of imposing a one-dimensional (1D) optical potential on the three-dimensional (3D) imbalanced Fermi gas. We show that this 1D optical potential can greatly enlarge the stability region of the FFLO state, relative to the case of the 3D Fermi gas without 1D periodic modulation. Furthermore, it is shown that there exists a direct connection between the center-of-mass momentum of the FFLO pairs and the wave vector of the optical potential. We propose that this concept can be used experimentally to resonantly enhance the stability region of the FFLO state.
\end{abstract}

DOI: 10.1103/PhysRevA.83.013606

PACS number(s): 03.75.Ss, 03.75.Hh, 74.25.Dw

\section{INTRODUCTION}

The study of superconducting and superfluid systems has recently attracted wide attention, among other things because of the realization of ultracold Fermi gases in optical lattices [1-4]. These systems can be considered as quantum simulators that can be used for probing fundamental problems in condensed-matter physics [5] (e.g., the search for exotic new phases in strongly magnetized superconductors). Ultracold Fermi gases offer important advantages over conventional superconductors, mainly because of their extensive tunability. In a superconductor, the number of spin-up and spin-down electrons is equal and the interaction strength is fixed. In ultracold Fermi gases, not only can the interaction strength be tuned by the use of Feshbach resonances [6-8], but also the population imbalance can be freely adapted. This experimental freedom has led to the study of a variety of new phenomena in imbalanced ultracold Fermi gases [9-13]. One fundamental question that is still not settled concerns the nature of the ground state of an imbalanced Fermi gas. When population imbalance between the spin-up and spin-down components is introduced into these systems, complete pairing is no longer possible. Clogston and Chandrasekhar suggested that above a critical imbalance, the superfluid system would undergo a transition into the normal state $[14,15]$. This effect has been observed experimentally by the MIT [9] and Rice [10] groups. However, their observations were not in exact agreement, and there still exists some controversy [16] about the exact nature of the phases of the superfluid system at high levels of imbalance. In 1964 Fulde and Ferell [17] and independently Larkin and Ovchinnikov [18] proposed that a superfluid system can accommodate population imbalance, by making a transition into a state with a finite center-of-mass

\footnotetext{
* On leave of absence from Department of Theoretical Physics, State University of Moldova, str. A. Mateevici 60, MD-2009 Kishinev, Republic of Moldova.
}

momentum (and thus a spatially modulated order parameter). This state is the so-called Fulde-Ferell-Larkin-Ovchinnikov state (FFLO state). Recently, there has been an ongoing theoretical search for this exotic state [19] (for a recent review, see Ref. [20]). In one dimension (1D), the FFLO state was predicted to exist in superconducting systems [21]. This work was confirmed numerically [22] and elaborated further through theoretical studies of the ground state and the phase diagram of a 1D Fermi gas [23-25]. Furthermore, it has been shown that the 1D analog of the FFLO state is stable in a large section of the crossover phase diagram from Bardeen-Cooper-Schrieffer (BCS) superfluidity to a Bose-Einstein condensate (BEC) of bound molecules [26], compared to the case of a three-dimensional (3D) Fermi gas. Although this 1D FFLO state has not yet been observed directly, a recent paper reports the experimental observation of density profiles that agree quantitatively with theoretical predictions at low temperature [27]. In three dimensions, however, the experimental observation of the FFLO state has so far remained elusive. One of the main reasons for this is that the FFLO state in three dimensions only occurs in a tiny section of the BCS-BEC crossover phase diagram [28,29]. This then raises the question: Is there a way to stabilize the FFLO state in a 3D Fermi gas? The purpose of this paper is twofold: First, we develop a path-integral description for a superfluid Fermi gas that can accommodate the FFLO state; second, we propose a method to stabilize the FFLO state through an optical potential. In two recent papers it was suggested to stabilize the FFLO state by the use of a 3D optical lattice [30,31]. In this paper we investigate the stabilizing effect of a 1D optical potential in order to investigate the interplay between the wave vector of the FFLO state and the wave vector of the laser that creates the optical potential. In the present work, the 1D optical potential provides a periodic modulation in one direction. We emphasize that we do not study the FFLO state in a 1D gas [32], but in a 3D gas with a superimposed 1D periodic potential. In Sec. II we describe the FFLO state in an imbalanced Fermi gas in 3D within the path-integral 
framework. As a test for the correctness of this description, we calculate the zero-temperature phase diagram for this system and compare our findings with recent theoretical results [28,29]. In Sec. III we investigate two models to account for the effect of a 1D optical potential. We show that the presence of such a potential leads to a substantial increase of the stability region of the FFLO state. Finally in Sec. IV we draw conclusions.

\section{PATH-INTEGRAL DESCRIPTION}

The partition sum of an imbalanced Fermi gas in 3D can be written as a path integral over the fermionic fields $\bar{\psi}_{\mathbf{k}, \omega_{n}, \sigma}$ and $\psi_{\mathbf{k}, \omega_{n}, \sigma}$ :

$$
\begin{aligned}
\mathcal{Z}= & \int \mathcal{D} \bar{\psi} \mathcal{D} \psi \exp \left(-\sum_{\mathbf{k}, n} \sum_{\sigma} \bar{\psi}_{\mathbf{k}, \omega_{n}, \sigma}\left(-i \omega_{n}+\mathbf{k}^{2}-\mu_{\sigma}\right)\right. \\
& \times \psi_{\mathbf{k}, \omega_{n}, \sigma}-\frac{g}{\beta L^{3}} \sum_{\mathbf{k}, n} \sum_{\mathbf{k}^{\prime}, n^{\prime}} \sum_{\mathbf{q}, m} \bar{\psi}_{(\mathbf{q} / 2)+\mathbf{k}, \Omega_{m}+\omega_{n}, \uparrow} \\
& \left.\times \bar{\psi}_{(\mathbf{q} / 2)-\mathbf{k}, \Omega_{m}-\omega_{n}, \downarrow} \psi_{(\mathbf{q} / 2)-\mathbf{k}^{\prime}, \Omega_{m}-\omega_{n^{\prime}}, \downarrow} \psi_{(\mathbf{q} / 2)+\mathbf{k}^{\prime}, \Omega_{m}+\omega_{n^{\prime}}, \uparrow}\right) .
\end{aligned}
$$

Here $\mathbf{k}$ is the wave vector, $\omega_{n}$ are the fermionic Matsubara frequencies, and $\sigma=\uparrow, \downarrow$ denote the two different hyperfine states. Furthermore, $\beta$ is the inverse temperature given by $1 / k_{B} T, L$ is the lateral size of the system, the chemical potential of a particle with spin $\sigma$ is denoted by $\mu_{\sigma}$, and $g$ is the renormalized interaction strength. We use units such that $\hbar=2 m=$ $E_{F}=1$. This partition sum (1) can be made more tractable by introducing the Hubbard-Stratonovich transformation, which decouples the fourth-order interaction term into second-order terms by introducing two auxiliary bosonic fields $\phi_{\mathbf{q}, \Omega_{m}}$ and $\bar{\phi}_{\mathbf{q}, \Omega_{m}}$, interpreted as the pair fields. As a first approximation, only the saddle point is taken into account in the path integral over the bosonic fields. To describe the FFLO state, we propose to use a saddle point at which the atomic pairs have a finite wave vector $\mathbf{Q}$ :

$$
\phi_{\mathbf{q}, \Omega_{m}}=\delta_{\mathbf{q}, \mathbf{Q}} \delta_{m, 0} \sqrt{\beta L^{3}} \Delta .
$$

By using this particular form of the saddle point, we choose to describe the FF state, which has an order parameter given by a plane wave $\sim e^{i \mathbf{Q} \cdot \mathbf{r}}$. It is also possible to describe the LO state, which is a superposition of two plane waves with wave vectors $\mathbf{Q}$ and $-\mathbf{Q}$. In this paper, we will not consider the LO state. For the remainder of this paper, the FF state is referred to as the FFLO state. When $\mathbf{Q}$ is set equal to zero in (2), the description of the normal superfluid is recovered [33]. In expression (2), the prefactor $\sqrt{\beta L^{3}}$ ensures that $\Delta$ has units of energy. By using (2), the fermionic fields can be integrated out in expression (1) for the partition function, leading to an effective saddle point (SP) action $\mathcal{S}_{\text {sp }}$, through

$$
\begin{aligned}
\mathcal{Z}_{\text {sp }} & =\exp \left(\sum_{\mathbf{k}, n} \ln \left[-\operatorname{det}\left(-\mathbb{G}_{\mathbf{k}, n}^{-1}\right)\right]+\frac{\beta L^{3}}{g}|\Delta|^{2}\right) \\
& =\exp \left(-\mathcal{S}_{\mathrm{sp}}\right),
\end{aligned}
$$

where $\mathbb{G}_{\mathbf{k}, n}^{-1}$ is the inverse Nambu propagator, which is given by

$$
\begin{aligned}
-\mathbb{G}_{\mathbf{k}, n}^{-1} & \\
\quad= & \left(\begin{array}{cc}
-i \omega_{n}+(\mathbf{Q} / 2+\mathbf{k})^{2}-\mu_{\uparrow} & -\Delta \\
-\Delta^{*} & -i \omega_{n}-(\mathbf{Q} / 2-\mathbf{k})^{2}+\mu_{\downarrow}
\end{array}\right) .
\end{aligned}
$$

Expression (3) can be simplified further by performing the sum over the Matsubara frequencies. Also, it is useful to express the results as a function of the total chemical potential $\mu=\left(\mu_{\uparrow}+\right.$ $\left.\mu_{\downarrow}\right) / 2$ and the imbalance chemical potential $\zeta=\left(\mu_{\uparrow}-\mu_{\downarrow}\right) / 2$. Furthermore, the interaction between particles is modeled with a two-body contact potential $V(r)=g \delta(r)$. The renormalized interaction strength $g$ can then be written as follows [34]:

$$
\frac{1}{g}=\frac{1}{8 \pi\left(k_{F} a_{s}\right)}-\sum_{\mathbf{k}} \frac{1}{2 k^{2}},
$$

where $a_{s}$ is the 3D $s$-wave scattering length. As a final step the continuum limit is taken, and a thermodynamic potential is associated with the effective action $\mathcal{S}_{\mathrm{sp}}=\beta \Omega_{\mathrm{sp}}$. This then results in

$$
\begin{aligned}
\frac{\Omega_{\mathrm{sp}}}{L^{3}}= & -\int \frac{d \mathbf{k}}{(2 \pi)^{3}}\left(\frac{1}{\beta} \ln \left[2 \cosh \left(\beta \zeta_{\mathbf{Q}, \mathbf{k}}\right)+2 \cosh \left(\beta E_{\mathbf{k}}\right)\right]\right. \\
& \left.-\xi_{\mathbf{Q}, \mathbf{k}}-\frac{|\Delta|^{2}}{2 k^{2}}\right)-\frac{|\Delta|^{2}}{8 \pi\left(k_{F} a_{s}\right)},
\end{aligned}
$$

where the following notations were introduced:

$$
\begin{gathered}
\xi_{\mathbf{Q}, \mathbf{k}}=k^{2}-\left(\mu-\frac{Q^{2}}{4}\right), \\
E_{\mathbf{k}}=\sqrt{\xi_{\mathbf{Q}, \mathbf{k}}^{2}+|\Delta|^{2}}, \\
\zeta_{\mathbf{Q}, \mathbf{k}}=\zeta+\mathbf{Q} \cdot \mathbf{k} .
\end{gathered}
$$

The resulting form of the thermodynamic potential (6) has a form that is similar to the original result for the homogeneous 3D Fermi gas derived by Iskin and Sá de Melo [35] and coincides with it for $Q \rightarrow 0$. In order to test the correctness of (6), the thermodynamic potential is used to calculate the zero-temperature phase diagram of an imbalanced Fermi gas in 3D. This can be done for a fixed number of particles or for fixed chemical potentials. To transform between these two descriptions, the number equations, given by

$$
\begin{gathered}
-\left.\frac{\partial \Omega_{\mathrm{sp}}}{\partial \mu}\right|_{\beta, V}=n=\frac{1}{3 \pi^{2}}, \\
-\left.\frac{\partial \Omega_{\mathrm{sp}}}{\partial \zeta}\right|_{\beta, V, \mu}=\delta n,
\end{gathered}
$$

must be solved. As an example, the phase diagram is calculated for a fixed density $n$ and for a fixed imbalance chemical potential $\zeta$. To do this, the first number equation (8) is solved, given values of $\zeta, \Delta$, and $Q$, to determine the chemical potential $\mu$. The set of values $(\mu, \zeta, \Delta$, and $Q$ ) is then substituted in the free energy $\mathcal{F}=\Omega_{\mathrm{sp}}+\mu n$. The minima in the free-energy landscape determine which state is the ground state of the system, for a given imbalance chemical potential $\zeta$ and a given interaction strength $1 / k_{F} a_{s}$. There are three local minima that can be identified in the free-energy 


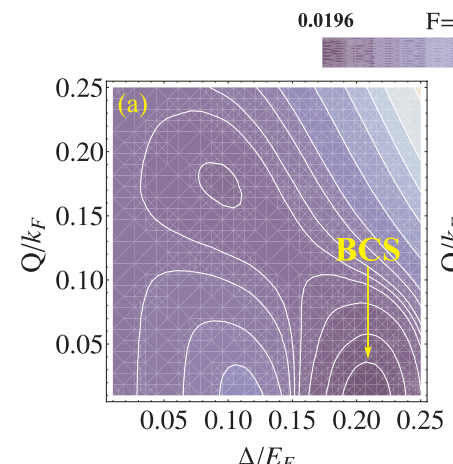

$\Delta / E_{F}$

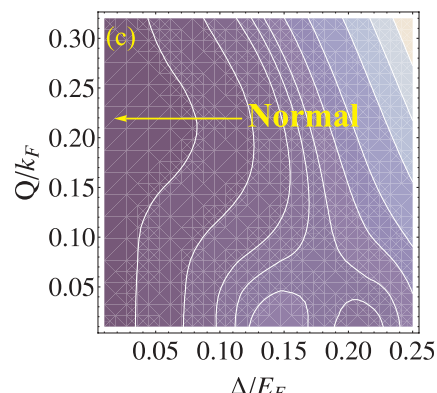

FIG. 1. (Color online) Free-energy landscape for an imbalanced Fermi gas in $3 \mathrm{D}$ at zero temperature, for different values of the imbalance chemical potential: (a) $\zeta / E_{F}=0.141$, (b) $\zeta / E_{F}=0.152$, and (c) $\zeta / E_{F}=0.164$. As the level of imbalance is increased, the competition between the local minima becomes apparent. Under the right circumstances, the FFLO state is the ground state of the system (b). The value of the interaction strength used here is $1 / k_{F} a_{S}=-1$.

landscape: the BCS state (spin-balanced superfluid) with $\Delta \neq 0, Q=0$; the FFLO state with $\Delta \neq 0, Q \neq 0$; and the normal state with $\Delta=0$. Figure 1 shows that the FFLO state can indeed be the ground state of an imbalanced Fermi gas in three dimensions (at zero temperature). In this figure, the free energy of the system is shown, as a function of the band gap $\Delta$ and the wave vector $\mathbf{Q}$, relative to the Fermi energy $E_{F}$ and the Fermi wave vector $k_{F}$, respectively.

For relatively small values of the imbalance chemical potential $\zeta$, the system is in the BCS ground state (a). When $\zeta$ increases, the system undergoes a first-order transition into the FFLO state (b). When the imbalance chemical potential increases further, the system continuously goes over into the normal state (c). Figure 1 shows these phase transitions for only one specific value of the interaction strength, near the BCS limit $\left(1 / k_{F} a_{S}=-1\right)$. However, since the first number equation is used to calculate the value of the chemical potential $\mu$, the description is also valid for the complete BCSBEC crossover regime. It must be noted that the mean-field approximation breaks down in the unitarity limit. However, the FFLO state is expected to form only in the BCS region of the BCS-BEC crossover, where we expect the mean-field description to give qualitatively correct results. The phase diagram of the system is shown in Fig. 2. This diagram shows that, theoretically, the FFLO state can be formed in an imbalanced Fermi gas in 3D, but since it only occurs on a tiny section of the phase diagram, it may be hard to

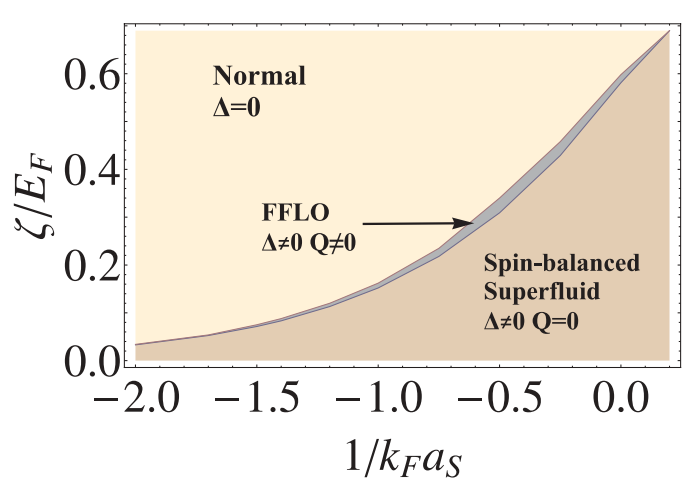

FIG. 2. (Color online) Phase diagram of an imbalanced Fermi gas in 3D at zero temperature, for fixed density $n$. As the imbalance chemical potential $\zeta$ increases, the system undergoes a first-order transition from a spin-balanced superfluid (BCS) to the FFLO state. Above a critical imbalance (which is dependent on the interaction strength), the FFLO state continuously goes over into the normal state.

observe this state experimentally. Our results coincide with recent theoretical results $[28,29]$.

\section{MODELING A ONE-DIMENSIONAL OPTICAL POTENTIAL}

As shown in Sec. II, the problem with detecting the FFLO state in an imbalanced Fermi gas in 3D is that it exists only in a relatively small section of the BCS-BEC phase diagram. In this section, we describe the 3D imbalanced Fermi gas in a 1D optical potential. There are two main reasons why such a potential can stabilize the FFLO state. The first reason is that in an imbalanced Fermi gas in 3D, the FFLO state can have a wave vector $\mathbf{Q}$ in an arbitrary direction. This freedom of choice leads to low-energy bosonic excitations, or Goldstone modes, which render the FFLO state unstable. In the presence of a 1D optical potential, however, it will be energetically favorable for the FFLO state to form in the direction of the optical potential. This will limit the choice for the wave vector $\mathbf{Q}$ to just one value, thus suppressing the Goldstone modes, which is expected to stabilize the FFLO state. The second reason is that the optical potential will enhance the 1D modulation of the FFLO order parameter. We therefore expect the enhancement to be largest when the wave vector of the FFLO state is equal to the wave vector of the optical potential. The present mean-field treatment does not include the effect of excitations such as the Goldstone modes, but it does include the energy lowering of the modulated order parameter due to the optical potential.

In this section we propose two approaches of modeling a 3D imbalanced Fermi gas in a 1D optical potential. In both approaches, the optical potential is described by using a modified dispersion relation. In Sec. III A we model the optical potential by introducing an anisotropic effective mass in the direction of the potential [36] (from here on this is supposed to be the $z$ direction). This approximation is valid when the Fermi energy of the system lies near the bottom of the lowest Bloch band (i.e., in the case of low density or a short-wavelength optical potential). In Sec. III B, we model the optical potential by treating the full lowest Bloch band in the tight-binding approximation [37-40]. This model is valid when the Fermi 
energy lies in the lowest Bloch band (otherwise more bands must be considered), but contrary to the first case, it does not need to lie at the bottom of the band. In both Secs. III A and III B the optical potential is supposed not to forbid tunneling, as this would inhibit the formation of the FFLO state. This implies that we will treat the imbalanced Fermi gas in 3D in a 1D optical potential as a three-dimensional system with a one-dimensional periodic modulation.

\section{A. Anisotropic effective mass}

In this section, the 1D optical potential is modeled through the use of a modified effective mass of the fermionic particles in the direction of the optical potential

$$
\varepsilon\left(k, k_{z}\right)=k^{2}+\frac{k_{z}^{2}}{2 m_{z}},
$$

where $m_{z}$ is the effective mass of the particles in the $z$ direction. Here and for the remainder of the paper, $k^{2}=k_{x}^{2}+k_{y}^{2}$ denotes the magnitude of the in-plane wave vector. This is the wave vector that lies perpendicular to the laser beam that creates the 1D optical potential. The derivation of the thermodynamic potential for this case is analogous to the derivation in Sec. II. In the present derivation, however, it is assumed that the FFLO state will form in the $z$ direction $\mathbf{Q}=(0,0, Q)$, because this is energetically favorable to other directions, due to the anisotropy introduced through (10). The resulting thermodynamic potential, in the limit for temperature going to zero, is given by

$$
\begin{aligned}
\frac{\Omega_{\mathrm{sp}}}{L^{3}}= & -\frac{1}{(2 \pi)^{2}} \int_{0}^{+\infty} d k k \int_{-\infty}^{+\infty} d k_{z} \\
& \times\left(\max \left[\left|\zeta_{\mathbf{k}, \mathbf{Q}}\right|, E_{\mathbf{k}}\right]-\xi_{\mathbf{k}}-\frac{|\Delta|^{2}}{2\left(k^{2}+\frac{k_{z}^{2}}{2 m_{z}}\right)}\right)-\frac{|\Delta|^{2}}{8 \pi\left(k_{F} a_{s}\right)},
\end{aligned}
$$

with the modified notations

$$
\begin{gathered}
\xi_{\mathbf{Q}, \mathbf{k}}=k^{2}+\frac{1}{2 m_{z}}\left(k_{z}^{2}+\frac{Q^{2}}{4}\right)-\mu, \\
E_{\mathbf{k}}=\sqrt{\xi_{\mathbf{Q}, \mathbf{k}}^{2}+|\Delta|^{2}}, \\
\zeta_{\mathbf{Q}, \mathbf{k}}=\frac{1}{2 m_{z}} k_{z} Q-\zeta .
\end{gathered}
$$

The number equations are still given by (8) and (9), but the density $n$ has changed to

$$
n=\frac{\sqrt{2 m_{z}}}{3 \pi^{2}}
$$

because of the modified dispersion relation (10). In the limit $m_{z} \rightarrow 1 / 2$, the thermodynamic potential (11) and the density (13) converge to the corresponding expressions in the case of an imbalanced Fermi gas in 3D, described in Sec. II. Expression (13) implies that when the effective mass $m_{z}$ changes, the density $n$ changes with it. It would be interesting, however, to compare the phase diagrams for Fermi gases with different effective masses at equal density. This can in fact be achieved, because we have found that the thermodynamic potential of the system with effective mass $m_{z}$ can be rescaled

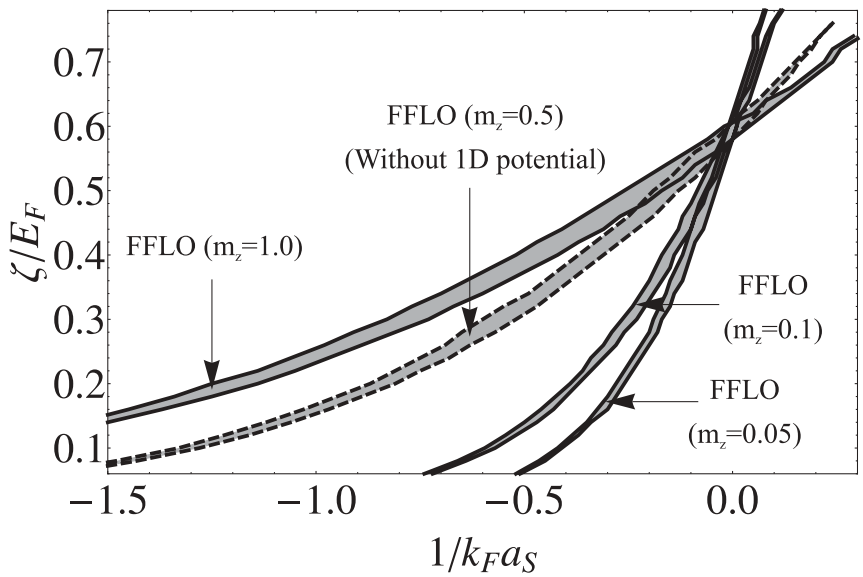

FIG. 3. Phase diagrams of 3D imbalanced Fermi gases in a 1D optical potential (at zero temperature), where the optical potential was modeled by altering the effective mass $m_{z}$ (in the $z$ direction) of the fermionic particles. Here, the phase diagrams are shown for different effective masses $m_{z}$. The gray regions indicate the FFLO phase. These phase diagrams are shown before rescaling according to (14), and hence the densities differ for the various systems with different effective masses. After rescaling, all FFLO regions map onto the case of $m_{z}=1 / 2$ (gray region bounded by dashed curves).

to the thermodynamic potential of the system with effective mass $m_{z}=1 / 2$ [Eq. (6)], using the following scaling relation:

$$
\begin{aligned}
& \Omega_{\mathrm{sp}}\left(\mu, \zeta, \Delta, Q, m_{z}, \frac{1}{a_{s}}\right) \\
& \quad=\sqrt{2 m_{z}} \Omega_{\mathrm{sp}}\left(\mu, \zeta, \Delta, \frac{Q}{\sqrt{2 m_{z}}}, \frac{1}{2}, \frac{1}{a_{s}} \frac{1}{\sqrt{2 m_{z}}}\right) .
\end{aligned}
$$

From a theoretical point of view, this rescaling property is efficient for calculations and gives a deeper insight into the role of the effective mass $m_{z}$. The main advantage, however, is that all physical properties can be studied at the same density. This property relates to experiment, because when an external potential is turned on, the effective mass is altered, but the average density will remain the same. The effect of changing $m_{z}$ on the BCS-BEC crossover phase diagram is shown in Fig. 3. This figure shows the FFLO phase boundaries of the imbalanced Fermi gas in 3D for different values of the effective mass $m_{z}$, before rescaling according to (14) (and hence at different densities). There is a tilting of the FFLO region about a fixed point at unitarity. Furthermore, there is an increase in the width of the FFLO region (relative to the abscissa) as the effective mass increases. By using the scaling relation (14), the phase diagrams in Fig. 3 can be rescaled to equal density. After rescaling, the phase diagrams for the different effective masses map onto the phase diagram of the imbalanced Fermi gas with isotropic effective mass $\left(m_{z}=1 / 2\right)$, described in Sec. II. From this we conclude that the FFLO state is not fundamentally influenced by an optical potential in which the Fermi energy lies near the bottom of the first Bloch band. This can be explained by the fact that no fundamental anisotropy is introduced into the system by altering the effective mass, because independent of the effective mass, the system can be 
scaled back to the case of the imbalanced Fermi gas, in which the effective mass equals $1 / 2$.

\section{B. Bloch dispersion}

In Sec. III A it was shown that a more fundamental anisotropy is needed, in order for the optical potential to have an effect on the FFLO state. In this section, we model a 1D optical potential in the tight-binding approximation, using the first Bloch band. For this purpose, the quadratic dispersion in the $z$ direction is replaced by a periodic dispersion [37-40]

$$
\varepsilon\left(k, k_{z}\right)=k^{2}+\delta\left[1-\cos \left(\frac{\pi k_{z}}{Q_{L}}\right)\right] \text {. }
$$

$$
\frac{\Omega_{\mathrm{sp}}}{L^{3}}=-\frac{1}{(2 \pi)^{2}} \int_{0}^{+\infty} d k k \int_{-Q_{L}}^{+Q_{L}} d k_{z}\left(\max \left[\left|\zeta_{k, Q}\right|, E_{\mathbf{k}}\right]-\xi_{\mathbf{k}}-\frac{\Delta^{2}}{2\left\{k^{2}+\delta\left[1-\cos \left(\frac{\pi k_{z}}{Q_{L}}\right)\right]\right\}}\right)-\frac{\Delta^{2}}{8 \pi\left(k_{F} a_{S}\right)}
$$

Here $Q_{L}$ is the wave vector of the optical potential and $\delta$ is a prefactor with units of energy, given by [40]

$$
\delta=8\left(\frac{V_{0}^{3} E_{R}}{\pi^{2}}\right)^{\frac{1}{4}} \exp \left(-2 \sqrt{\frac{V_{0}}{E_{R}}}\right)
$$

where $V_{0}$ is the depth of the potential and $E_{R}$ is the recoil energy given by $E_{R}=2 \pi^{2} \hbar^{2} / m \lambda^{2}$, with $\lambda$ the wavelength of the optical potential and $m$ the mass of the fermionic particles. In the limit for small $k_{z}$, expression (15) simplifies to $(10)$, with

$$
m_{z}=\frac{Q_{L}^{2}}{\delta \pi^{2}}
$$

Given the new dispersion (15), the thermodynamic potential for this system can be calculated. The result is with the following notations:

$$
\begin{gathered}
\xi_{\mathbf{Q}, \mathbf{k}}=k^{2}+\delta\left[1-\cos \left(\frac{\pi}{2} \frac{Q}{Q_{L}}\right) \cos \left(\frac{\pi k_{z}}{Q_{L}}\right)\right]-\mu \\
E_{\mathbf{k}}=\sqrt{\left\{k^{2}+\delta\left[1-\cos \left(\frac{\pi}{2} \frac{Q}{Q_{L}}\right) \cos \left(\frac{\pi k_{z}}{Q_{L}}\right)\right]-\mu\right\}^{2}+\Delta^{2}} \\
\zeta_{\mathbf{Q}, \mathbf{k}}=\zeta-\delta \sin \left(\frac{\pi}{2} \frac{Q}{Q_{L}}\right) \sin \left(\frac{\pi k_{z}}{Q_{L}}\right)
\end{gathered}
$$

It can easily be shown that expression (18) is equal to the corresponding thermodynamic potential (11) of the anisotropic effective mass case, in the limit $Q_{L} \rightarrow \infty, \delta \rightarrow \infty$ with $m_{z}$ held constant, according to (17). The two number equations again are given by (8) and (9) and the density $n$ can be calculated using the general expression

$$
n=2 \int \frac{d \mathbf{k}}{(2 \pi)^{3}} \Theta\left(1-\left\{k^{2}+\delta\left[1-\cos \left(\frac{\pi k_{z}}{Q_{L}}\right)\right]\right\}\right),
$$

which yields

$$
n=\left\{\begin{array}{c}
\frac{Q_{L}}{2 \pi^{2}}(1-\delta), \quad 1 \geqslant 2 \delta, \\
\frac{Q_{L}}{2 \pi^{3}}\left[(1-\delta) \arccos \left(\frac{\delta-1}{\delta}\right)+\delta \sqrt{1-\left(\frac{\delta-1}{\delta}\right)^{2}}\right], \quad 1<2 \delta .
\end{array}\right.
$$

Here it must be noted that our derivation is exact only if $E_{F}<2 \delta$, because otherwise more than one Bloch band must be considered. As in the previous sections, the phase diagram for this system can be constructed by studying the local minima of the free energy. Figure 4 shows a comparison between the phase diagram of an imbalanced Fermi gas in 3D and the phase diagram of an imbalanced Fermi gas in 3D subject to a $1 \mathrm{D}$ optical potential, modeled in the tight-binding approximation and using the first Bloch band.

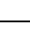

This figure shows that the FFLO region is enlarged by a factor of 3 to 6 , due to the stabilizing effect of the 1D optical potential. Figure 4 further shows that at $\zeta / E_{F} \approx 0.48$ a transition point occurs, at which the FFLO region reaches a maximum width (relative to the abscissa), and narrows quickly for larger values of $\zeta$. This effect finds its origin in the magnitude of the wave vector of the FFLO pairs $Q_{\text {FFLO }}$. When the imbalance chemical potential $\zeta$ increases, $Q_{\mathrm{FFLO}}$ likewise increases to accommodate the widening gap between the Fermi surfaces of the two spin species. At a certain level of imbalance

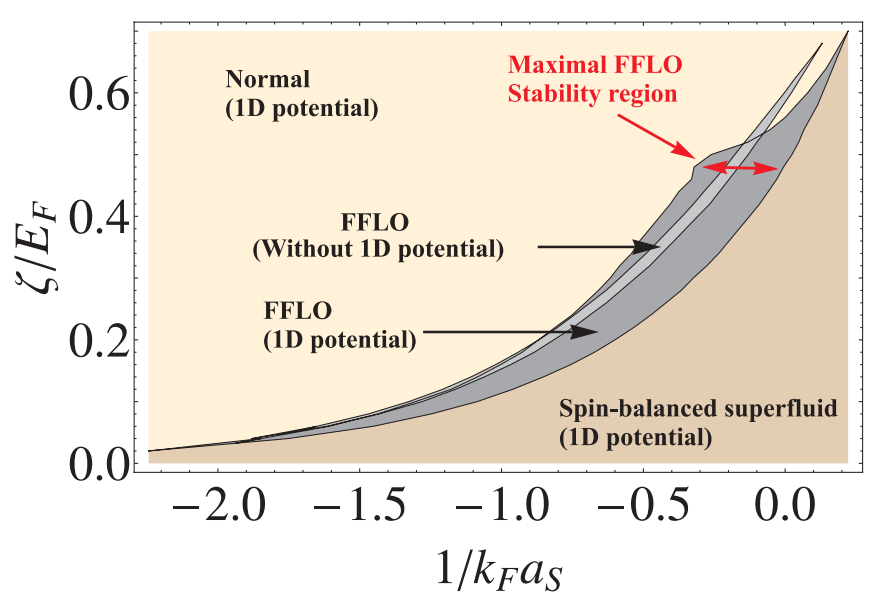

FIG. 4. (Color online) Comparison between the phase diagram of an imbalanced Fermi gas in 3D and an imbalanced Fermi gas in $3 \mathrm{D}$ subjected to a $1 \mathrm{D}$ optical potential (with $\delta=0.5$ and $Q_{L}=1.2$ ), modeled in the tight-binding approximation, using the first Bloch band. Both phase diagrams are at zero temperature. The stabilizing effect of the optical potential enlarges the FFLO region by a factor of 3 to 6 when compared to the case of the imbalanced Fermi gas without optical potential. When $\zeta / E_{F} \approx 0.48$, the width of the FFLO region is maximal (for this specific choice of $\delta$ and $Q_{L}$ ). This resonant enhancement of the FFLO region occurs when the wave vector of the FFLO pairs is equal to the wave vector of the optical potential. 


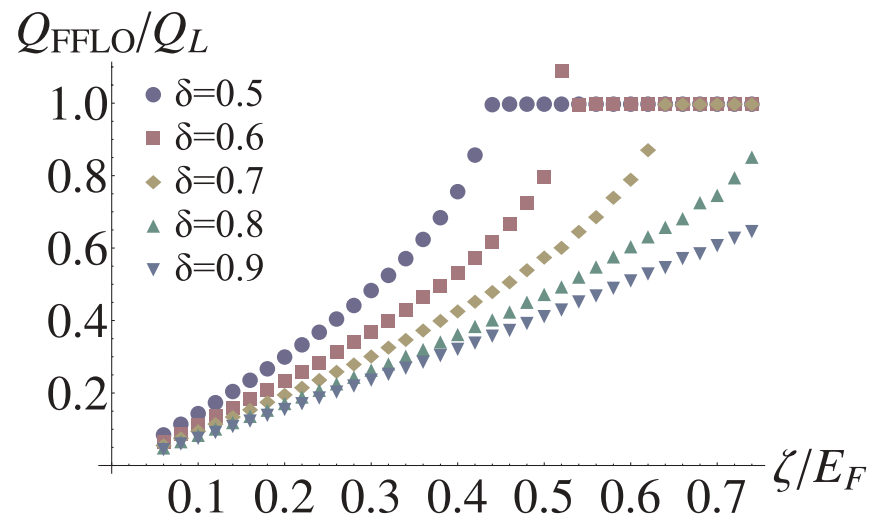

FIG. 5. (Color online) The wave vector of the FFLO state $Q_{\text {FFLO, }}$ relative to the wave vector of the $1 \mathrm{D}$ optical potential $Q_{L}$, as a function of the imbalance chemical potential $\zeta$ (relative to the Fermi energy $\left.E_{F}\right)$. The value of $Q_{\text {FFLO }}$ increases with increasing imbalance, until it becomes equal to the value of the wave vector of the optical potential. When imbalance increases further, $Q_{\text {FFLO }}$ retains the constant value $Q_{L}$. The single overshoot point for $\delta=0.6$ is probably a numerical inaccuracy.

(in the case of Fig. 4, at $\zeta / E_{F} \approx 0.48$ ), $Q_{\mathrm{FFLO}}$ equals the wave vector of the optical potential $Q_{L}$. At this point, the FFLO state is optimally enhanced, because the spatial modulation of the FFLO state is equal to the spatial modulation of the optical potential. This results in a maximal width of the FFLO region. When $\zeta$ increases further, $Q_{\text {FFLO }}$ retains the constant value $Q_{L}$, and is not able to grow any further. This effect is shown in Fig. 5. Hence, we can conclude that, although the imbalance has increased further, the optical potential forces the FFLO state into a state in which the form of the FFLO order parameter matches the form of the optical potential. This results in a narrowing of the FFLO region, because the wave vector of the FFLO state is no longer sufficiently large to bridge the gap between the Fermi surfaces of the spin-up and spin-down particles. The value of the transition point, at which $Q_{\text {FFLO }}$ becomes equal to $Q_{L}$, roughly increases linearly with the value of $\delta$, as shown in Figs. 6(a)-6(c). Qualitatively, this is because the rate of change of the FFLO wave vector $Q_{\text {FFLO }}$, with increasing imbalance chemical potential $\zeta$, decreases when $\delta$ becomes larger. This means that $\zeta$ must be larger (compared to the case of lower $\delta$ ) for $Q_{\text {FFLO }}$ to reach the limiting wave vector of the optical potential $Q_{L}$. The advantage of this resonant enhancement of the FFLO state is that, for a given level of imbalance, an optimal stability region for the FFLO state can be created, simply by tuning the wavelength of the $1 \mathrm{D}$ optical potential. It should be noted that, when considering an imbalance chemical potential $\zeta$ smaller than $0.48, \delta$ must become smaller than 0.5 and more bands must be taken into account in order for our description to be exact. We do not treat this case in the present paper. One could raise the question of whether the FFLO state at the resonant point, at which the FFLO wave vector equals the wave vector of the optical lattice, reduces to the trivial phase of a superfluid that has acquired Bragg peaks in momentum space [41]. In our current treatment, however, we consider a saddle point at which the order parameter acquires one specific wave vector $Q$. This implies that in our case, we have either a pure BCS

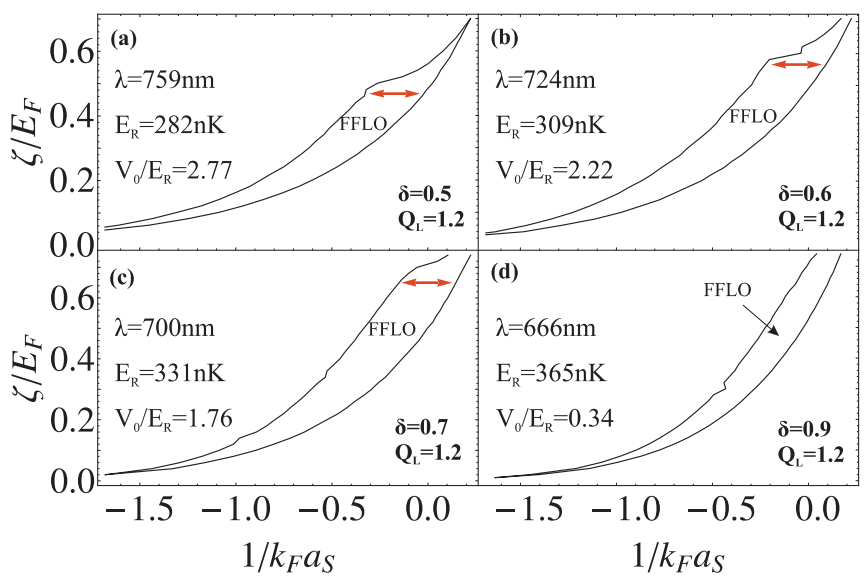

FIG. 6. (Color online) Several phase diagrams of an imbalanced superfluid Fermi gas in 3D subjected to a 1D optical potential, for increasing values of $\delta$. The value of the imbalance chemical potential $\zeta$ at which the maximal FFLO region occurs roughly scales linearly with the value of $\delta$. For each level of imbalance, an optimal FFLO region can be found, by tuning the wavelength of the optical potential. Panel (d) shows that in the limit of small potential depth $V_{0}$, the FFLO region decreases. This agrees with the intuition that when $V_{0} \rightarrow 0$ the case of the effective mass, described in Sec. III A, should be retrieved.

state with $Q=0$ or a pure FFLO state with $Q \neq 0$. Therefore, in the current formalism we do not consider a superposition of the two states, and whenever the wave vector $Q$ is nonzero, the corresponding state is FFLO by definition. Figure 6(d) illustrates that when the potential depth becomes very small, the width of the FFLO region decreases. This is as one would expect, because in the limit of very small potential depth, the description in terms of an effective mass of Sec. III A should be obtained.

To obtain a more direct link with experimental parameter values, we convert our units back to SI units for the three situations depicted in Fig. 6. A possible choice of atoms that we considered is ${ }^{40} \mathrm{~K}$ atoms in a one-dimensional harmonic trap, with a density of $10^{13} \mathrm{~cm}^{-3}$. For instance, the case with $\delta=0.6$ and $Q_{L}=1.2$ corresponds to the case of an optical potential with wavelength equal to $724 \mathrm{~nm}$, a recoil energy of $309 \mathrm{nK}$, and an optical potential depth of $V_{0} / E_{R} \approx 2.22$. The numerical values for these experimental parameters in the case of $\delta=0.5, \delta=0.7$, and $\delta=0.9$ are depicted in Figs. 6(a), $6(\mathrm{c})$, and 6(d), respectively. For the illustrative cases of Fig. 6 we use $Q_{L}=1.2$, but theoretically, any choice of $Q_{L}$ was possible because we found that the wave vector of the optical potential $Q_{L}$ acts as a scaling parameter, according to the following scaling relation:

$$
\begin{aligned}
& \Omega_{\mathrm{sp}}\left(\mu, \zeta, \Delta, Q, \delta, \alpha Q_{L}, \frac{1}{k_{F} a_{S}}\right) \\
& \quad=\alpha \Omega_{\mathrm{sp}}\left(\mu, \zeta, \Delta, \frac{1}{\alpha} Q, \delta, Q_{L}, \frac{1}{\alpha} \frac{1}{k_{F} a_{S}}\right) .
\end{aligned}
$$

In principle this means that we can vary $Q_{L}$ from zero to infinity. However, there exist some limitations on this parameter. First, there is a lower limit for $Q_{L}$ because below a certain value of $Q_{L}$ no value of the optical potential depth 
$V_{0}$ can satisfy Eq. (16), given values for $\delta$ and for the recoil energy $E_{R}$. Second, when the depth of the optical potential becomes too large, the Fermi gas will become a system of weakly coupled two-dimensional Fermi gases. This means that our formalism, which assumed that the Fermi gas was 3D, is no longer valid. During the course of our work, Loh and Trivedi published their results on the LO state in a 3D cubic lattice [30]. They found that in a 3D cubic lattice, the LO state was more stable than the FF state. Since we already find a substantial increase in the FF state using a 1D optical potential, we expect that the effect on the LO state will be similar or larger. It would be interesting to apply our 1D-potential scheme also to the LO case.

\section{CONCLUSIONS}

We have described the FFLO state in an imbalanced Fermi gas in 3D within the path-integral framework, by choosing a suitable saddle point at which the atomic pairs have a finite center-of-mass momentum. As a platform to address the case of a 3D imbalanced Fermi gas in a 1D optical potential and to validate our path-integral description, we rederived the zero-temperature phase diagram for an imbalanced Fermi gas in $3 \mathrm{D}$. For this case, our results coincide with recent theoretical results. As a proposal to stabilize the FFLO state, we have studied an imbalanced 3D Fermi gas in a 1D optical potential. This potential was modeled in two different ways. For the first model, in which we considered anisotropic effective masses, we have found that this model is a rescaling of the case of a 3D imbalanced Fermi gas with isotropic effective mass. In the second model, we described the effect of the 1D optical potential using the first Bloch band in the tight-binding approximation. In this case we have found a substantial increase in the stability region of the FFLO state, as compared to the case of the 3D Fermi gas without the 1D optical potential. Related results were recently found in the case of a 3D cubic optical lattice [30,31]. The advantage of our 1D optical potential scheme, compared to a 3D cubic optical lattice, is that it allows us to find an optimal stability configuration for the FFLO state with a given level of imbalance, by tuning the wavelength of the optical potential. This resonant enhancement of the FFLO region occurs when the wave vector of the FFLO pairs is equal to the wave vector of the optical potential. This tunability makes a $1 \mathrm{D}$ optical potential a suitable experimental configuration for the stabilization of the FFLO state. We propose, therefore, that this concept can facilitate the experimental observation of the FFLO state in an imbalanced Fermi gas in 3D.

\section{ACKNOWLEDGMENTS}

The authors would like to thank Vladimir Gladilin and Fons Brosens for fruitful discussions. This work was supported by Fonds voor Wetenschappelijk Onderzoek-Vlaanderen (FWO-V) projects G.0356.06, G.0370.09N, G.0180.09N, and G.0365.08.
[1] W. Hofstetter, J. I. Cirac, P. Zoller, E. Demler, and M. D. Lukin, Phys. Rev. Lett. 89, 220407 (2002).

[2] G. Modugno, F. Ferlaino, R. Heidemann, G. Roati, and M. Inguscio, Phys. Rev. A 68, 011601(R) (2003).

[3] M. Köhl, H. Moritz, T. Stöferle, K. Günter, and T. Esslinger, Phys. Rev. Lett. 94, 080403 (2005).

[4] J. K. Chin, D. E. Miller, Y. Liu, C. Stan, W. Setiawan, C. Sanner, K. Xu, and W. Ketterle, Nature (London) 443, 961 (2006).

[5] I. Bloch, Nat. Phys. 1, 23 (2005).

[6] E. Timmermans, P. Tommasini, R. Côté, M. Hussein, and A. Kerman, Phys. Rep. 315, 199 (1999).

[7] A. V. Andreev, V. Gurarie, and L. Radzihovsky, Phys. Rev. Lett. 93, 130402 (2004).

[8] N. Strohmaier, Y. Takasu, K. Günter, R. Jördens, M. Köhl, H. Moritz, and T. Esslinger, Phys. Rev. Lett. 99, 220601 (2007).

[9] M. W. Zwierlein, A. Schirotzek, C. H. Schunck, and W. Ketterle, Science 311, 492 (2006); M. W. Zwierlein, C. H. Schunck, A. Schirotzek, and W. Ketterle, Nature (London) 442, 54 (2006).

[10] G. B. Partridge, W. Li, R. I. Kamar, Y. A. Liao, and R. G. Hulet, Science 311, 503 (2006).

[11] Y. Shin, M. W. Zwierlein, C. H. Schunck, A. Schirotzek, and W. Ketterle, Phys. Rev. Lett. 97, 030401 (2006).

[12] G. B. Partridge, W. Li, Y. A. Liao, R. G. Hulet, M. Haque, and H. T. C. Stoof, Phys. Rev. Lett. 97, 190407 (2006).

[13] C. H. Schunck, Y. Shin, A. Schirotzek, M. W. Zwierlein, and W. Ketterle, Science 316, 867 (2007).

[14] B. S. Chandrasekhar, Appl. Phys. Lett. 1, 7 (1962).
[15] A. M. Clogston, Phys. Rev. Lett. 9, 266 (1962).

[16] F. Chevy, Physics 2, 48 (2009).

[17] P. Fulde and R. A. Ferrell, Phys. Rev. 135, A550 (1964).

[18] A. I. Larkin and Y. N. Ovchinnikov, Zh. Eksp. Teor. Fiz. 47, 1136 (1964) [Sov. Phys. JETP 20, 762 (1965)].

[19] T. Mizushima, K. Machida, and M. Ichioka, Phys. Rev. Lett. 94, 060404 (2005); D. E. Sheehy and L. Radzihovsky, ibid. 96, 060401 (2006); J. Kinnunen, L. M. Jensen, and P. Törmä, ibid. 96, 110403 (2006); K. Machida, T. Mizushima, and M. Ichioka, ibid. 97, 120407 (2006); P. Castorina, M. Grasso, M. Oertel, M. Urban, and D. Zappalà, Phys. Rev. A 72, 025601 (2005); N. Yoshida and S.-K. Yip, ibid. 75, 063601 (2007); W. Zhang and L.-M. Duan, ibid. 76, 042710 (2007); T. K. Koponen, T. Paananen, J.-P. Martikainen, M. R. Bakhtiari, and P. Törmä, New J. Phys. 10, 045014 (2008).

[20] L. Radzihovsky and D. E. Sheehy, Rep. Prog. Phys. 73, 076501 (2010).

[21] K. Yang, Phys. Rev. B 63, 140511(R) (2001).

[22] A. E. Feiguin and F. Heidrich-Meisner, Phys. Rev. B 76, 220508(R) (2007).

[23] H. Hu, X. J. Liu, and P. D. Drummond, Phys. Rev. Lett. 98, 070403 (2007).

[24] G. Orso, Phys. Rev. Lett. 98, 070402 (2007).

[25] M. M. Parish, S. K. Baur, E. J. Mueller, and D. A. Huse, Phys. Rev. Lett. 99, 250403 (2007).

[26] F. Heidrich-Meisner, A. E. Feiguin, U. Schollwöck, and W. Zwerger, Phys. Rev. A 81, 023629 (2010). 
[27] Y. Liao, A. S. C. Rittner, T. Paprotta, W. Li, G. B. Partridge, R. G. Hulet, S. K. Baur, and E. J. Mueller, Nature (London) 467, 567 (2010).

[28] H. Hu and X. J. Liu, Phys. Rev. A 73, 051603(R) (2006).

[29] D. E. Sheehy and L. Radzihovsky, Ann. Phys. (NY) 322, 1790 (2007).

[30] Y. L. Loh and N. Trivedi, Phys. Rev. Lett. 104, 165302 (2010).

[31] T. K. Koponen, T. Paananen, J.-P. Martikainen, and P. Törmä, Phys. Rev. Lett. 99, 120403 (2007).

[32] K. B. Gubbels, M. W. J. Romans, and H. T. C. Stoof, Phys. Rev. Lett. 97, 210402 (2006); T.-L. Dao, M. Ferrero, A. Georges, M. Capone, and O. Parcollet, ibid. 101, 236405 (2008); C.-C. Chien, Q. Chen, Y. He, and K. Levin, ibid. 97, 090402 (2006); 98, 110404 (2007).

[33] J. Tempere, M. Wouters, and J. T. Devreese, Phys. Rev. B 75, 184526 (2007).
[34] C. A. R. Sá de Melo, M. Randeria, and J. R. Engelbrecht, Phys. Rev. Lett. 71, 3202 (1993).

[35] M. Iskin and C. A. R. Sá de Melo, Phys. Rev. Lett. 97, 100404 (2006).

[36] M. Ben Dahan, E. Peik, J. Reichel, Y. Castin, and C. Salomon, Phys. Rev. Lett. 76, 4508 (1996).

[37] M. Krämer, C. Menotti, L. Pitaevskii, and S. Stringari, Eur. Phys. J. D 27, 247 (2003).

[38] L. Pezzè, L. Pitaevskii, A. Smerzi, S. Stringari, G. Modugno, E. de Mirandes, F. Ferlaino, H. Ott, G. Roati, and M. Inguscio, Phys. Rev. Lett. 93, 120401 (2004).

[39] W. Zwerger, J. Opt. B 5, S9 (2003).

[40] A. Koetsier, D. B. M. Dickerscheid, and H. T. C. Stoof, Phys. Rev. A 74, 033621 (2006).

[41] M. K. Oberthaler, R. Abfalterer, S. Bernet, J. Schmiedmayer, and A. Zeilinger, Phys. Rev. Lett. 77, 4980 (1996). 